\title{
SOME CHARACTERIZATIONS OF NONLINEAR FIRST ORDER DIFFERENTIAL EQUATIONS ON UNBOUNDED INTERVALS
}

\author{
BAPURAO C. DHAGE
}

Abstract. In this article, we discuss the existence as well as global attractivity and ultimate positivity of solutions for a certain nonlinear first order ordinary differential equation on the unbounded intervals of real line via a classical fixed point theorem of Schauder (Smart [12, page 15]). Our hypotheses and claims have also been explained with the help of two realizations under some natural conditions.

Mathematics subject classification (2010): 47H10, 34A60.

Keywords and phrases: nonlinear differential equation, fixed point theorem, attractive solutions, ultimately positive solutions.

\section{REFERENCES}

[1] J. BANAS AND B. RZEPKA, An application of a measure of noncompactness in the study of asymptotic stability, Appl. Math. Letters, 16 (2003), 1-6.

[2] J. BANAS AND B. C. DHAGE, Global asymptotic stability of solutions of a integral equations, Nonlinear Analysis, 69 (2008), 1945-1952.

[3] T. A. Burton AND B. Zhang, Fixed points and stability of an integral equation: nonuniqueness, Appl. Math. Letters, 17 (2004), 839-846.

[4] T. A. Burton And T. Furumochi, A note on stability by Schauder's theorem, Funkcialaj Ekvacioj, 44 (2001), 73-82.

[5] B. C. DHAGE, Asymptotic stability of nonlinear integral equations via measures of noncompactness, Comm. Appl. Nonlinear Anal., 15, 2 (2008), 89-101.

[6] B. C. DhaGe, Local asymptotic attractivity for nonlinear quadratic integral equations, Nonlinear Analysis, 70, 5 (2009), 1912-1922.

[7] B. C. DHAGE, Global attractivity results for nonlinear integral equations via a Krasnoselskii type fixed point theorem, Nonlinear Analysis, 70 (2009), 2485-2493.

[8] B. C. DHAGE, Attractivity and positivity results for nonlinear functional integral equations via measures of noncompactness, Differ. Equ. Appl., 2, 3 (2010), 00-00.

[9] B. C. Dhage And V. LAKshmikantham, Quadratic perturbations of periodic boundary value problems of second order ordinary differential equations, Nonlinear Analysis, to appear.

[10] A. Granas And J. Dugundj, Fixed Point Theory, Springer Verlag, New York, 2003.

[11] X. HU AND J. YAN, The global attractivity and asymptotic stability of solution of a nonlinear integral equation, J. Math. Anal. Appl., 321 (2006), 147-156.

[12] D. R. Smart, Fixed Point Theorems, Cambridge Univ. Press, Cambridge 1974. 